\title{
Capillary Driven Two-Phase Flow Dynamics in Nonpatterned and Patterned Microchannels
}

\author{
A. Vafaie $^{1,2}$ and M. Tahmasebipour ${ }^{1,2,3 *}$ \\ ${ }^{1}$ Faculty of New Sciences and Technologies, University of Tehran, Tehran, Iran \\ *E-mail: tahmasebipour@ut.ac.ir \\ Phone: +98 21 86093187; Fax: +98 2186093187 \\ ${ }^{2}$ Micro/Nano-fabrication Technologies Development Laboratory, Faculty of New \\ sciences \& Technologies, University of Tehran, Tehran, Iran \\ ${ }^{3}$ Micro/Nanofluidic Systems Laboratory, Faculty of New sciences \& Technologies, \\ University of Tehran, Tehran, Iran
}

\begin{abstract}
Study of the behavior of fluids inside micro and nanochannels has become particularly important with the extensive advances in micro- and nanofluidic systems. Capillary filling is a phenomenon that occurs in microchannels when the fluid is in contact with the channel walls. This phenomenon can be controlled by introducing certain characteristics in the channel walls and these channels are used in specific applications such as microreactors and pressure-sensitive switches. In this paper new insights about some effective parameters in the capillary are provided by which it is possible to increase or decrease the fluid's velocity or even stop its motion at a specific point in the microchannel. The influence of different regimes governed the capillary action on the fluid's velocity is studied. Furthermore, the effect of introducing certain obstacles on the microchannel wall on the capillary action and its relation with the contact angle of fluid is investigated. 2D FEM capillary simulation for three different fluids at contact angles of 30,50 and $60^{\circ}$ showed that at $30^{\circ}$ and $50^{\circ}$ fluid passes the obstacle and at $60^{\circ}$ remains pinned at the obstacle. Finally, certain grooves on the channel walls are used to increase fluid's velocity. Results showed that the grooves increase fluid velocity by $15 \%$.
\end{abstract}

Keywords: capillary dynamics; capillary regimes; patterned microchannel; two-phase flow.

\section{INTRODUCTION}

The capillary filling phenomenon is observed when a liquid contacts the hydrophilic walls of a channel. In fact, the capillary filling action occurs due to surface tension of the fluid as well as the attraction force between the hydrophilic surface and the fluid. Recent technological advances in the fabrication of microfluidic devices have led to special applications of capillary filling in microchannels and given rise to various applications for this phenomenon including micro heat exchangers, lab-on-a-chip systems, nanoimprinting, and micro-reactors. DNA, protein, and virus separation is another application of nanofluidic systems where capillary filling plays a direct role.

Due to the importance of the capillary filling and its direct effect in micro and nanofluidic systems, various researches have been conducted in recent years in this field. Although the governing equations of capillary action dynamics were proposed in 1921, the parameters influencing capillary dynamics are still being studied due to the existing 
shortfalls in this regard. Majhy et al. [1] studied the dynamics of capillary flow of oil in an open superoleophilic channel. Bakli et al. [2] presented a study on dynamics of water passing through a porous surface with nanometric voids, and a comprehensive model was presented for describing the forces that resist water penetration. This model expressed the capillary length in terms of fluid contact angle and fluid acceleration. In the same context, Acquaroli et al. [3] conducted an experimental study on capillary filling dynamics inside a nanoporous silicon layer and compared the experimental results with those obtained from theoretical equations expressing capillary dynamics in terms of ambient pressure and capillary pressure. Nan et al. [4] studied the capillary filling speed of ferrofluids through nanofluidic PDMS channels. It was observed that, under different temperature and magnetic field conditions, the fluid contact angle changed with fluid speed as it passed through the nanochannel. Chakraborty [5] presented a semi-analytical model for capillary filling of blood under electro-osmotic forces. This model was expressed in terms of some effective parameters such as the voltage applied to the fluid, percentage of red blood cells in the fluid and fluid contact angle. Berli et al. [6] performed an analytical study on capillary filling of non-Newtonian fluids passing through conical channels. In this study channel geometry and fluid viscosity were recognized as factors that influenced capillary dynamics. Schneider et al. [7] studied capillary in closed-end nanochannels, in which air is trapped at the channel end (that gave rise to a different capillary mechanism in such channels). Through identification of the parameters affecting the fluid flow in microchannels, fluid flow can be controlled and used for specific purposes. For example, by reducing or increasing the channel width (termed as "global" and "local" resistances), Berthier et al. [8] reduced fluid flow velocity to a desired level and controlled it. In addition, Safavieh et al. [9] studied complicated microfluidic circuits which can be used to control and adjust the flow of several consecutive fluids with different velocities and return flows. Likewise, Wi et al. [10] increased the cooling efficiency of microscale heatcooling by setting a hydrophobic and hydrophilic wettability pattern on a microchannel surface.

Capillary filling creates a negative pressure in the liquid phase. This negative pressure can introduce curvature at polymeric channels and nanochannels, increasing their resistance to fluid penetration. In addition, as some of the existing channel fabrication methods lack the necessary precision for creating fully smooth channel walls, roughness of the channel walls fabricated using these methods is greater than expected. For this reason, it is essential to study the effect of such surface roughness on the fluid behavior. In addition, some researches indicated that capillary filling in a channel can be controlled by inserting certain obstacles and structures on the inner walls of the channel, and that this characteristic can be used for making different devices. For example, such microchannels can be used to manufacture micro-reactors and pressure-sensitive switches. Olanrewaju et al. [11] reviewed capillary microfluidic systems in which patterned microchannels were used in different parts of them. Dewangan et al. [12] studied the pressure driven incompressible flow through a microchannel which has a sinusoidal wall pattern. Qi and $\mathrm{Ng}$ [13] presented an analytical model developed for rotating electroosmotic flow in a narrow slit channel, of which the wall shape and surface potential vary in direction of applied fields. Likewise, such patterned microchannels can be used in cell separation application. In this field, Bui et al. [14] developed a PDMS microchannel for protein collection purpose. By patterning the internal walls of a microchannel with a simple structure, Kusumaatmaja et al. [15] increased fluid velocity through the channel and, in addition, built one-way microchannels. Nayak et al. [16] proposed an electroosmotic flow mixing enhancement in a sufficiently long nanochannel 
with a rectangular nonconducting obstacle mounted on the microchannel wall. Similarly, Chibbaro et al. [17] studied the effect of cubic obstacles on the capillary filling via three simulation methods (CFD, MD, and LBE) and compared obtained numerical results with those obtained from the Concus-Finn method. By controlling the contact angles between the fluid and channel walls, Huang et al. [18] modified the geometric shape of the fluid surface and fluid velocity for specific applications.

In the present research, fluid pressure distribution, and fluid velocity fields in nonpatterned and patterned microchannels have been studied. At first, the capillary filling mechanism in a 2D microchannel (without obstacles) was modeled, and its resulting pressure and velocity field profiles were studied. Upon examining the respective velocity fields, a different capillary filling dynamics regime was observed at the initial moments of the capillary filling. Then, the capillary filling mechanism in a microchannel with a rectangular obstacle on its inner walls surface was studied for three different fluids with contact angles of 30,50 and $60^{\circ}$, and their corresponding velocity variations were obtained in terms of their related capillary heights. Finally, the effect of creating grooves into the inner walls of the microchannel, aimed at increasing capillary filling speed, as well as the effect of such grooves on the common interface of the two fluids was examined.

\section{THEORY}

When a fluid contacts with a channel with hydrophilic walls, the capillary force $\left(F_{s}\right.$, Equation 1) overcomes the existing viscous force $\left(F_{v}\right.$, Equation 2$)$, pulling the fluid into the channel. Based on this, the capillary length $(l)$ versus time $(t)$, called the Washburn equation, is obtained (Equation 3).

$$
\begin{aligned}
& F_{s}=2 \gamma \cos \left(\theta_{e}\right) \\
& F_{v}=\frac{12 \mu l(d l / d t)}{H^{2}} \\
& l=\sqrt{\frac{\gamma H \cos \left(\theta_{e}\right) t}{3 \mu}}
\end{aligned}
$$

Where $\gamma$ is surface tension, $\mu$ is the fluid dynamic viscosity, $\theta_{e}$ is the contact angle between the fluid and the channel walls, and $H$ is the channel width. In this equation, the following assumptions are made: channel depth is infinite, channel walls are fully smooth, and the inertial and gravity effects are negligible [15]. As can be observed in this equation, capillary length is proportional to square root of time.

However, the Washburn equation has certain shortcomings. For example, the fluid initial velocity calculated from this equation is infinity (which is not logical). Consequently, in a model presented for different mechanisms governing fluid flow [19], two regimes were introduced for capillary: the inviscid regime and the Washburn regime. In the inviscid regime, viscous forces are negligible and fluid flow occurs due to the equilibrium state created between inertial and capillary forces. The term $\tau_{c}$ is defined based on the fluid characteristics and channel dimensions. For, $t \sim \tau_{c}$ the capillary dynamics lies in the inviscid regime where we have $l \sim t(\gamma / \rho R)^{0.5}(R=$ capillary radius $)$. For, $t » \tau_{c}$, however, the capillary dynamics is governed by the Washburn equation (Equation 3 ). 
There are different methods for tracing the interface between the fluids involved in the capillary action. One is the level set method [20] where flows are assumed laminar. In this method, the Navier-Stokes equations (conservation of momentum) and the continuity equation (conservation of mass) are solved. Then, the interface between the fluids is traced by solving the transfer equation for the level set function. This method implements the existing relation for incompressible fluid in the Navier-Stokes equations (Equations 4 and 5).

$$
\rho\left(\frac{\partial u}{\partial t}\right)+\rho(u \cdot \nabla) u=\nabla \cdot\left[-p l+\mu\left(\nabla u+\nabla u^{T}\right)\right]+F_{g}+F_{s t}
$$

$\nabla . u=0$

where, $\rho$ is density, $u$ is fluid flow velocity, $p$ is pressure, $I$ is the identity matrix, $\mu$ is dynamic viscosity, $F_{g}$ is the gravitational force, and $F_{s t}$ is surface tension. In the level set method, Equation 6 is added to the above equations.

$$
\frac{\partial \varphi}{\partial t}+u . \nabla \varphi=\gamma_{r} \nabla \cdot\left(\varepsilon \nabla \varphi-\varphi(1-\varphi) \frac{\nabla \varphi}{|\nabla \varphi|}\right)
$$

where $\gamma_{r}$ is the reinitialization parameter, $\varepsilon$ is interface thickness controlling parameter, and $\varphi$ is the level set function. Now, density can be defined as a function of the level set function as in Eq. (7).

$$
\rho=\rho_{1}+\left(\rho_{2}-\rho_{1}\right) \varphi
$$

In addition, dynamic viscosity is defined as Eq. (8).

$$
\mu=\mu_{1}+\left(\mu_{2}-\mu_{1}\right) \varphi
$$

where, $\rho_{1}$ and $\rho_{2}$ are constant densities of Fluids 1 and 2, respectively, and $\mu_{1}$ and $\mu_{2}$ are dynamic viscosities of Fluid 1 and Fluid 2, respectively. Here, Fluid 1 represents a region where $\varphi<0.5$ and Fluid 2 corresponds to a region defined as $\varphi>0.5$. By using this method, we can trace the interface between the fluids.

In Figure 1, if obstacles are placed on the inner walls of the channel, the capillary action in the channel faces limitations that are independent of the obstacle and the channel dimensions. According to the Concus-Finn criteria [21,22], if the contact angle of the inlet liquid is greater than $90^{\circ}-\alpha$, then the fluid stops upon reaching the obstacle. In the case where a rectangular obstacle is placed inside the channel, we expect - according to the same rule - that at contact angles of $\theta_{e}<45^{\circ}$, the fluid flow would be separated from the obstacle after encountering it and that the capillary length would increase, whereas for $\theta_{e}>45^{\circ}$, the fluid would stop upon encountering the obstacle. 


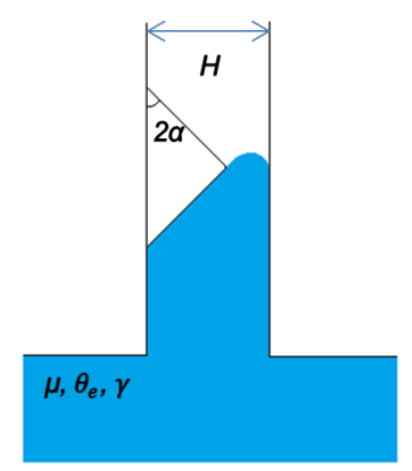

Figure 1. Schematic form of the capillary effect in a channel with an obstacle.

\section{CAPILLARY FILING DYNAMICS IN MICROCHANNELS WITHOUT OBSTACLE}

In this study, COMSOL software was used for finite element modelling of capillary filling. Schematic form of the modelled channel and the reservoir are shown in Figure 2. Table 1 lists the channel and reservoir dimensions as well as the properties of the two fluids involved in the process. Both fluids used in the modelling are assumed incompressible.

Table 1. Channel and reservoir dimensions and the characteristics of the fluids involved in the capillary filling.

\begin{tabular}{ccccccc}
$\begin{array}{c}\text { Contact } \\
\text { angle } \\
\left(\theta_{e}\right)\end{array}$ & $\begin{array}{c}\text { Reservoir } \\
\text { dimension }\end{array}$ & $\begin{array}{c}\text { Channel } \\
\text { dimension }\end{array}$ & $\begin{array}{c}\text { Fluid 1 } \\
\text { viscosity }\end{array}$ & $\begin{array}{c}\text { Fluid 2 } \\
\text { viscosity }\end{array}$ & $\begin{array}{c}\text { Fluid 1 } \\
\text { density }\end{array}$ & $\begin{array}{c}\text { Fluid 2 } \\
\text { density }\end{array}$ \\
\hline 60 & $\begin{array}{c}200 \times 480 \\
(\mu \mathrm{m})\end{array}$ & $\begin{array}{c}640 \times 50 \\
(\mu \mathrm{m})\end{array}$ & $\begin{array}{c}0.83 \\
(\mathrm{~Pa} . \mathrm{s})\end{array}$ & $\begin{array}{c}0.03 \\
(\mathrm{~Pa} . \mathrm{s})\end{array}$ & $\begin{array}{c}1.0 \\
\left(\mathrm{~kg} / \mathrm{m}^{3}\right)\end{array}$ & $\begin{array}{c}1.0 \\
\left(\mathrm{~kg} / \mathrm{m}^{3}\right)\end{array}$ \\
\hline
\end{tabular}

First, the capillary filling in a microchannel with fully smooth walls with the geometry shown in Figure 2 was modelled. The inlet, outlet, and the initial interface between the fluids are shown in this figure. The initial conditions for the fluids included zero pressure and velocity. Zero pressure boundary condition was imposed at the channel outlet. The channel walls were assumed to be hydrophilic, and the capillary was assumed to occur at a wet angle of $60^{\circ}$. In addition, the gravitational effects were applied to both fluids. 


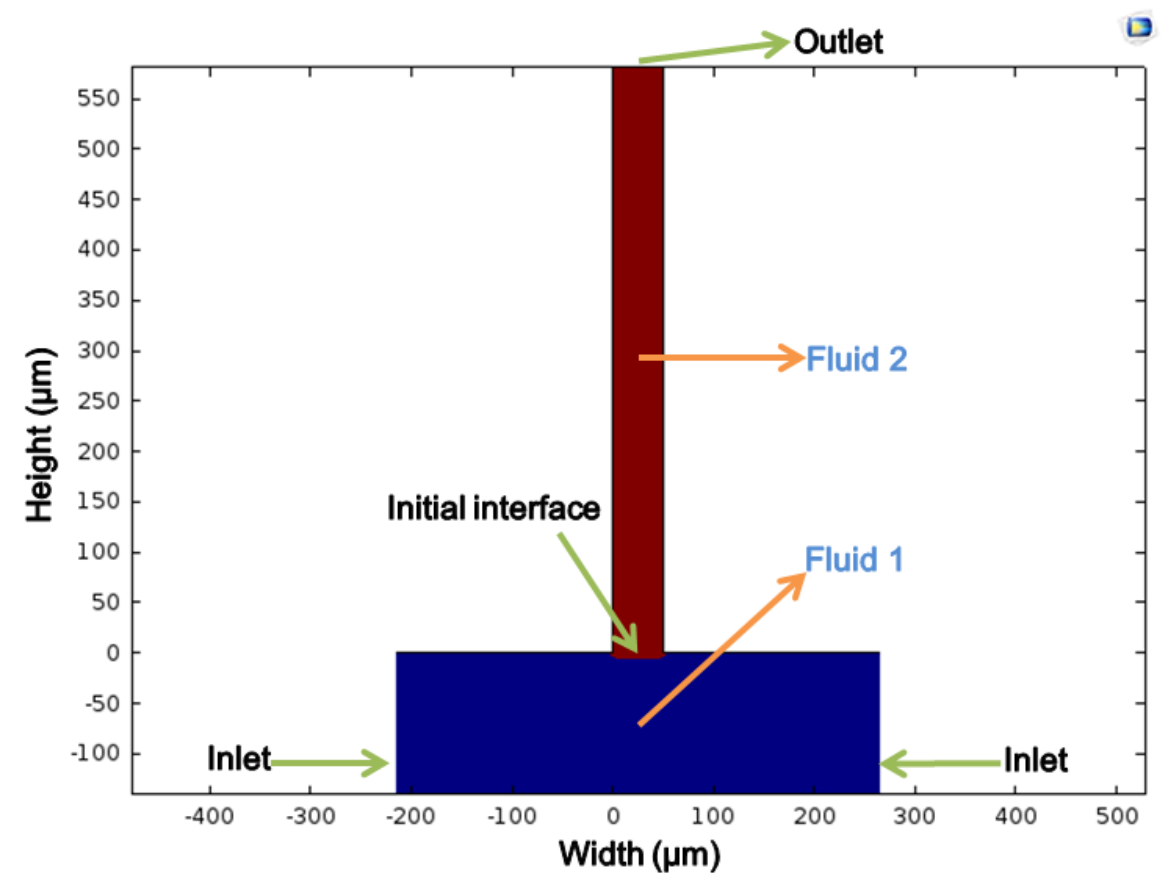

Figure 2. 2D model of channel and reservoir used in the capillary filling analysis.

During the capillary action, a negative pressure is created in Fluid 1 at the inlet to the channel, causing the fluid to enter the channel. This is confirmed by the pressure profile obtained from the modelling (Figure 3). In this figure, the pressure distribution in Fluids 1 and 2 are depicted at 0.01, 0.02, and 0.04 seconds. As can be observed, the negative pressure region moved upward as time passed, increasing the capillary height and displacing the fluids interface as shown with the yellow line. The negative pressure introduced near the interface acts in combination with the surface tension force to move Fluid 1 through the defined inlets towards the mouth of the channel, and from there towards the outlet.
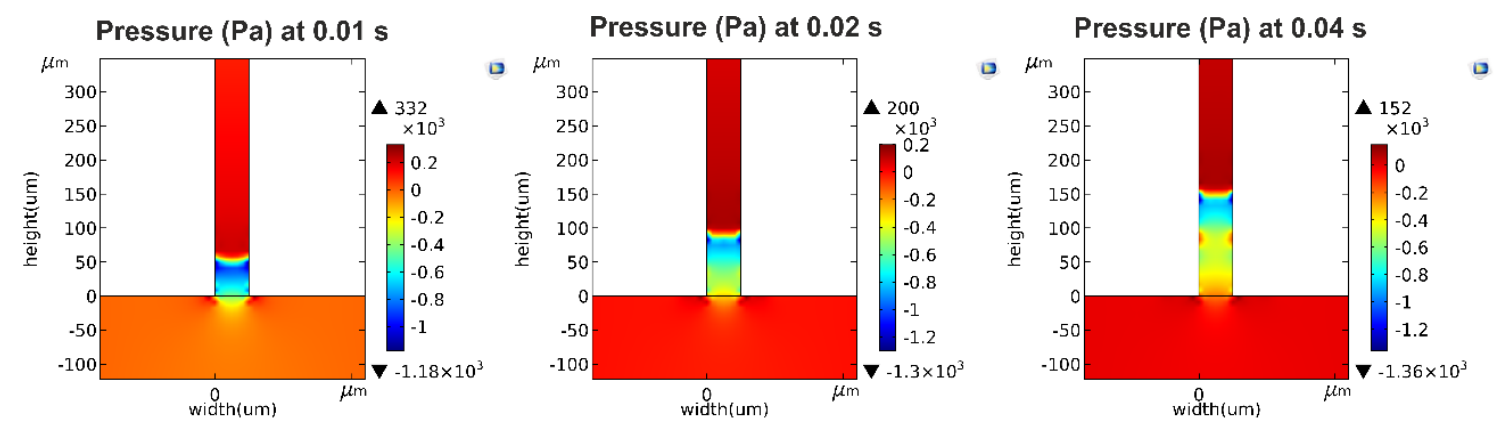

Figure 3. Pressure distribution in fluids 1 and 2 at $0.01,0.02$, and $0.04 \mathrm{s.}$

Figure 4 shows the velocity fields at $0,0.01,0.02$, and 0.04 seconds for the two fluids. The directions of the arrows and their size represent the respective fluids velocities directions and magnitudes, respectively. As can be observed, the mean fluid velocity within the channel decreased (as expected) by time. This is confirmed by the Eq. (3) that the fluid velocity is expected to gradually decrease. 

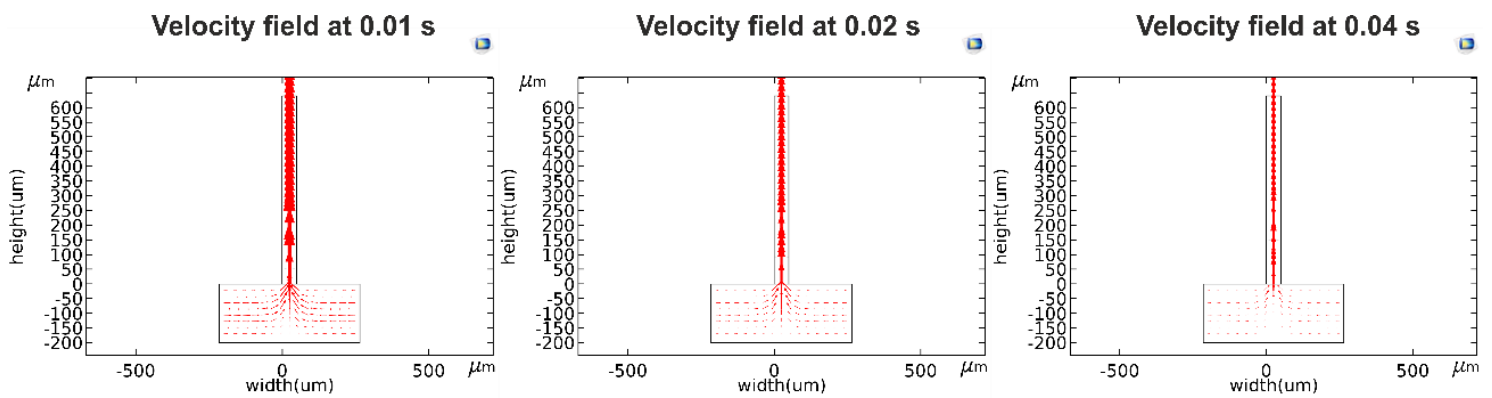

Figure 4. Velocity fields obtained for the fluids at $0.01,0.02$, and $0.04 \mathrm{~s}$.

As observed in Figure 4, the capillary action velocity was decreased during the studied period, which agrees with the Washburn regime. To investigate the existence of other regimes, we examined the capillary dynamics at shorter intervals. If, upon the start of the capillary effect, a constant mean velocity is obtained for the fluid within a short interval, then we can assume an inviscid regime for the fluid. Figure 5 shows the vertical velocity profiles obtained at the intersection of the channel and the reservoir at $0,0.002$, and 0.004 seconds.

In Figure 5, the maximum velocity (approximately $0.009 \mathrm{~m} / \mathrm{s}$ ) at the channel mouth occurred near the walls at $0 \mathrm{~s}$. The vertical component of the fluid velocity increased gradually by time at the centreline of the channel, until at $t=0.004 \mathrm{~s}$, a velocity profile in the shape of a full crescent was obtained across the channel. Although the fluid velocity obviously increased at $0.004 \mathrm{~s}$ (compared with $t=0.002 \mathrm{~s}$ ), the average vertical fluid velocities obtained between $t=0$ to $t=0.08 \mathrm{~s}$ have been used for comparing the total initial velocities of the fluids. Based on this, the vertical mean capillary velocity was calculated as a function of time (Figure 6). According to the Figure 6, in the interval between $0 \mathrm{~s}$ and $0.01 \mathrm{~s}$, capillary speed is linearly proportional to time. Therefore, it is concluded that capillary length in the same interval would be proportional to the square of time. Thus, a regime other than the Washburn regime [19] is observed during the first moments of the capillary action.

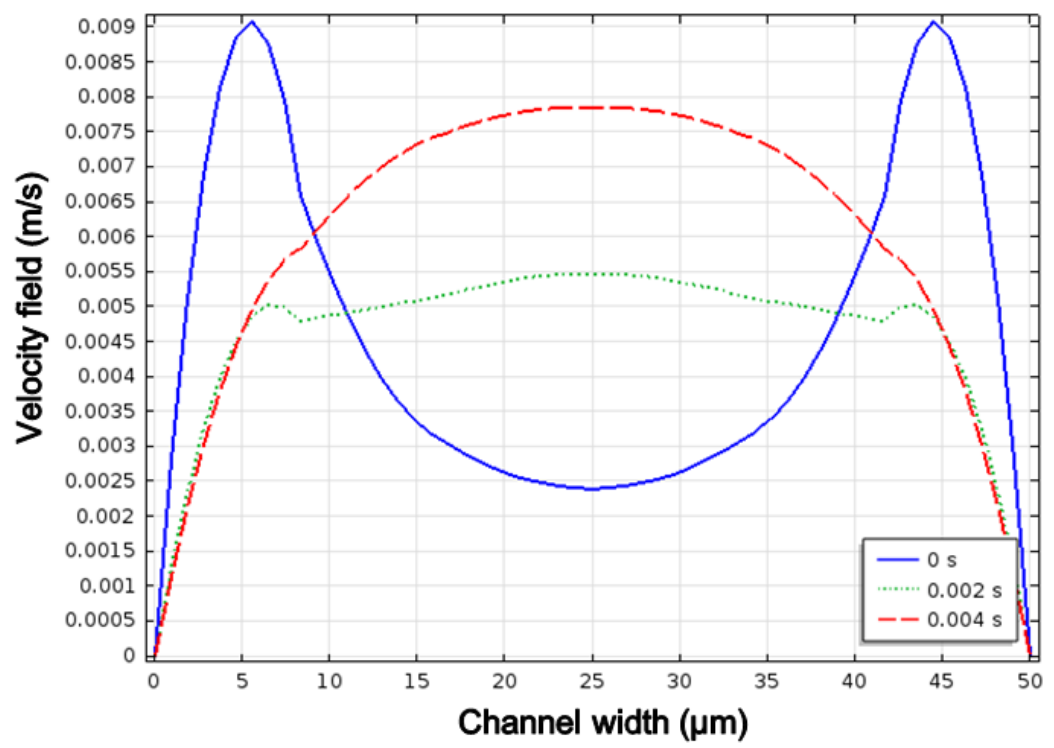

Figure 5. Vertical velocity profiles obtained at the channel-reservoir interface at 0 , 0.002 , and $0.004 \mathrm{~s}$. 


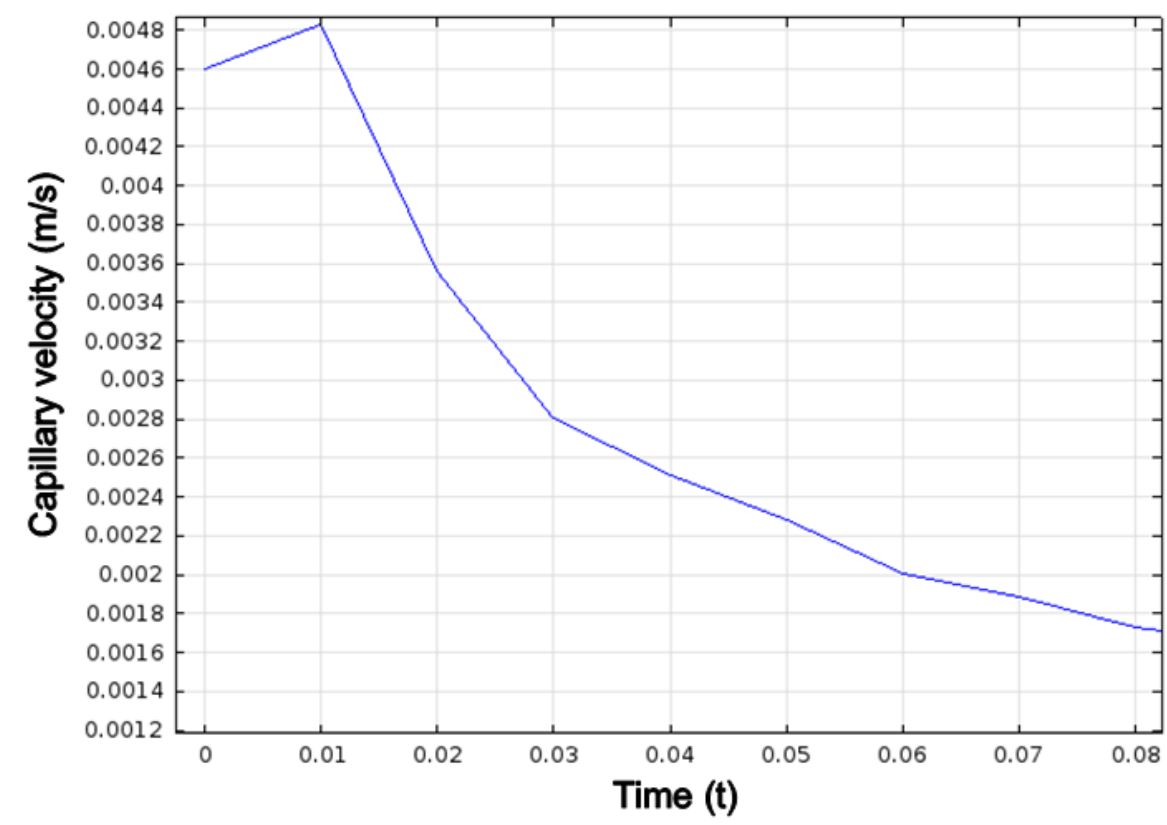

Figure 6. Capillary velocity versus time obtained for a channel with smooth walls.

Figure 7 shows the variations of capillary height with time obtained using the FEM modelling and the Washburn equation for the studied microchannel. As can be observed, although inertial and gravity effects were disregarded in the Washburn equation, there is a good agreement between the results obtained from this modelling and the theoretical results. Note that, as in the previous diagram, the theoretical curve for $t<0.01 \mathrm{~s}$ lies above the one obtained from modelling, indicating that a different regime governs the capillary action at the beginning of the capillary process.

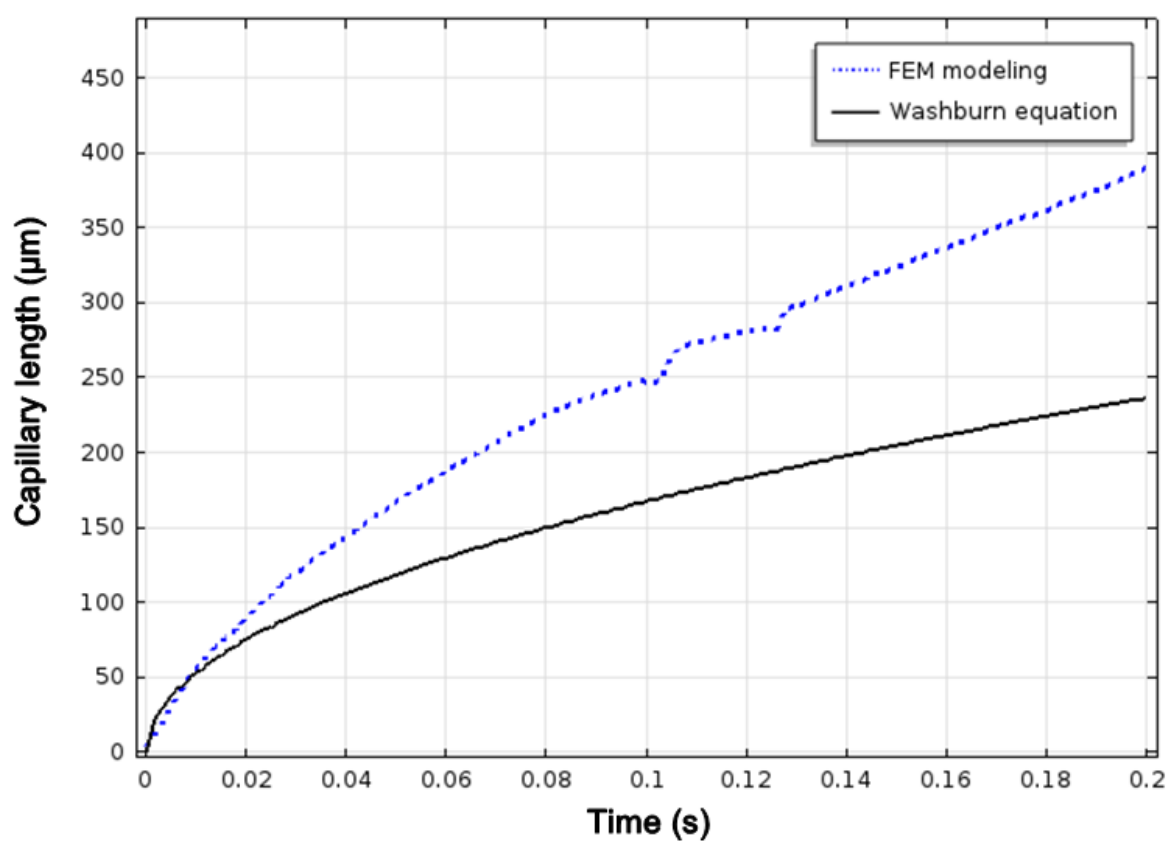

Figure 7. Capillary length against time obtained from theory and modelling for a microchannel with smooth walls. 


\section{CAPILLARY FILING DYNAMICS IN MICROCHANNELS WITHOUT OBSTACLE}

The capillary filling in a microchannel with a rectangular obstacle (with height of $40 \mu \mathrm{m}$ and width of $10 \mu \mathrm{m}$ ) on its inner wall was studied using the finite element analysis. The obstacle was placed at a height of $40 \mu \mathrm{m}$ from the intersection of the reservoir and the channel. Figure 8 shows the reservoir, microchannel, and obstacle geometries.

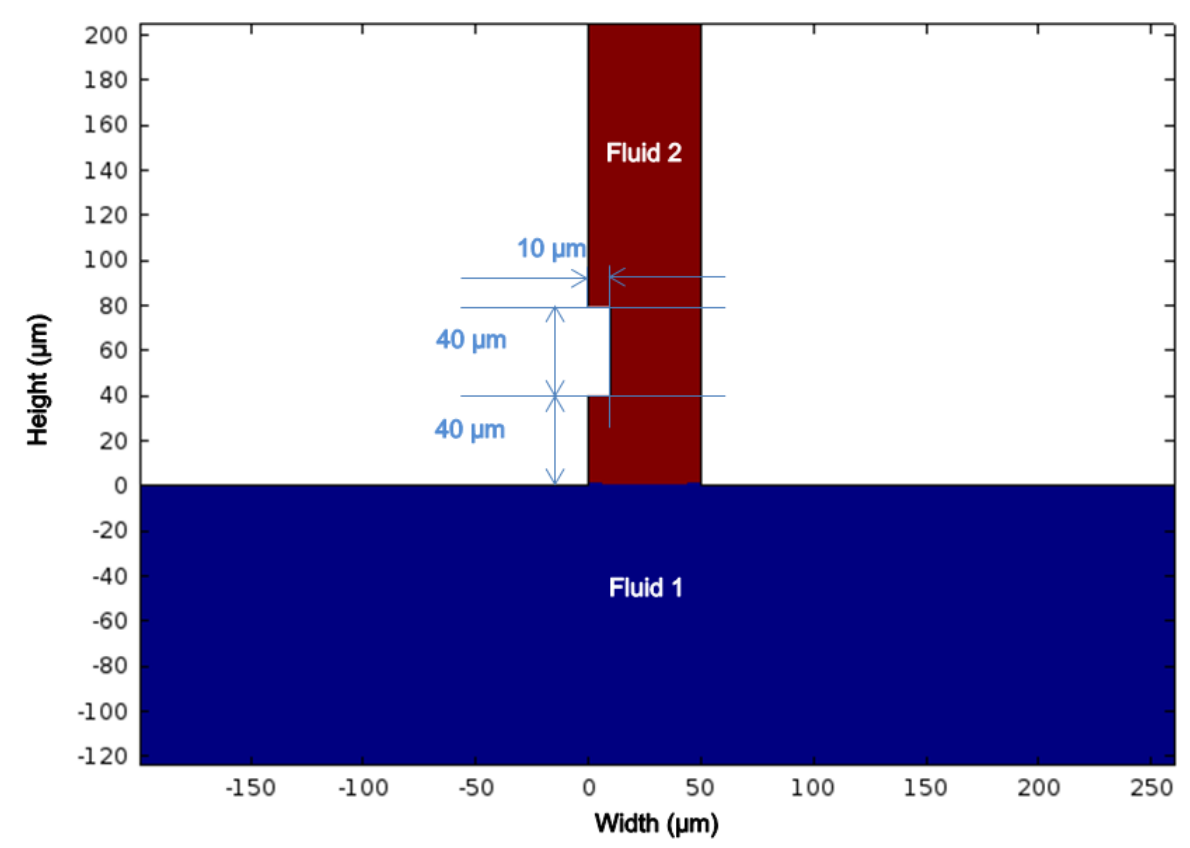

Figure 8. Reservoir, microchannel, and obstacle geometries.

According to the Gibbs rule $[21,22]$, for $\theta_{e}>45^{\circ}$, the capillary action in the channel is stopped upon the fluid encountering the obstacle. Accordingly, the capillary effect for three contact angles of 30,50 and $60^{\circ}$ was examined in this study. The capillary velocity versus capillary length is shown in Figure 9 for the three contact angles. As can be observed, the capillary action in these fluids followed a similar trend until a capillary height of $80 \mu \mathrm{m}$ (representing the obstacle end), with their only difference in the fluid velocity according to the Eq. (3), which shows that a fluid with a smaller contact angle exhibits a greater capillary velocity. 


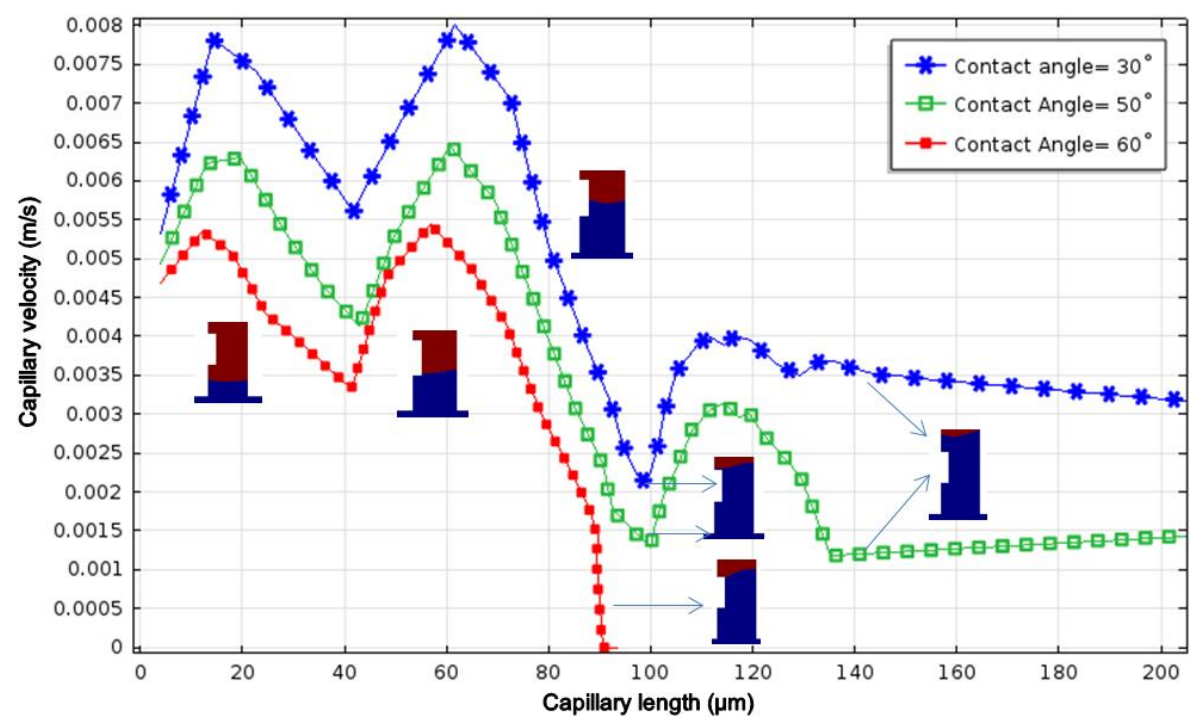

Figure 9. Capillary velocity versus capillary length during the capillary filling action.

Similar to the results obtained in Section 3, the capillary velocity increased in the capillary length of 0 to $10 \mu \mathrm{m}$ (i.e., the initial moments of the capillary action) due to the existence of a different regime. Thereafter, as it entered the Washburn regime, fluid velocity decreased. This trend was continued until the height of 40 microns in the channel when Fluid 1 is encountered the obstacle. Since then, similar to what happened at the start of the capillary, the velocity increased. The increase in velocity was continued until a height of $60 \mu \mathrm{m}$ at the middle of the obstacle. Thereafter, the capillary action was governed again by the Washburn regime until the end of the obstacle was reached. Upon encountering the obstacle end, a considerable reduction was observed in the velocity because - upon reaching the obstacle end at a height of $80 \mu \mathrm{m}$ - the fluid is remained pinned to the obstacle edge. The difference in the capillary velocity trends between three fluids was started after this point.

As shown in Figure 9, at a contact angle of $60^{\circ}$, the fluid remained pinned to the obstacle edge until its velocity reached zero, whereas the fluid having a contact angle of $30^{\circ}$ was separated from the obstacle edge, increasing the capillary height. Therefore, the fluids which have contact angles of 30 and $60^{\circ}$ follow the Gibbs rule. However, the Gibbs rule is violated at a contact angle of $50^{\circ}$ and the capillary continues. Under conditions similar to those used in the modelling process in this study, the Lattice-Boltzmann simulation predicts that the capillary action must stop at $\theta_{e}=50^{\circ}$ in accordance with the Gibbs rule [15]. However, the MD simulation produced results similar to those obtained in the present study where the fluid height continues to increase in the channel at $\theta_{e}=50^{\circ}$ [17].

\section{CAPILLARY FILLING DYNAMICS IN GROOVE-PATTERNED CHANNELS}

In this research, the effect of different patterns (Figure 10) on capillary velocity was studied using 3D finite element simulation. To this end, four microchannels with different cross sections (shown in Figs. 10 and 11) were modelled. Two grooveless channels were modelled for comparison purposes. The four channels had identical lengths and widths of 200 and $30 \mu \mathrm{m}$, respectively. 

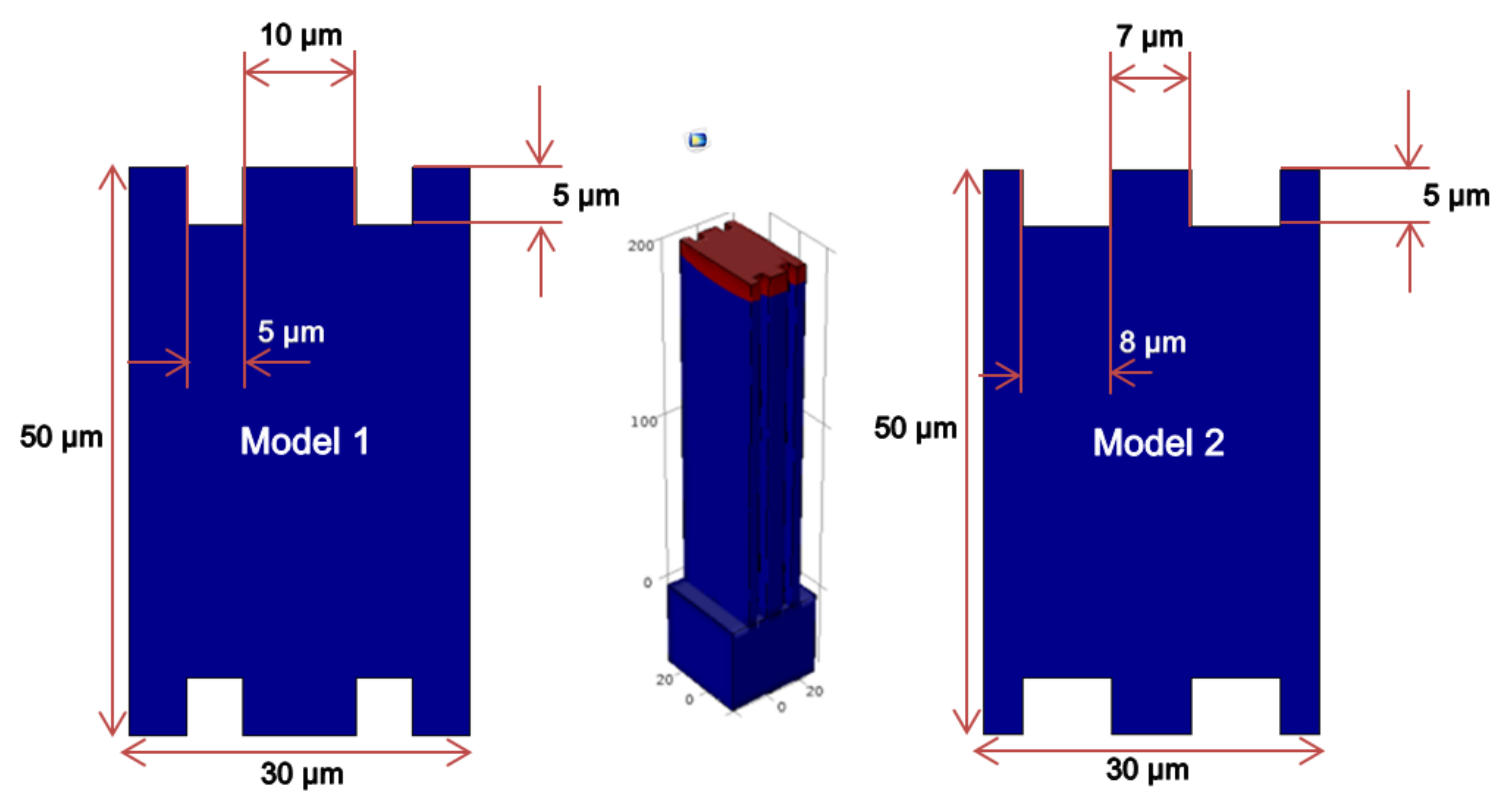

Figure 10. 3D model of the grooved microchannels with their respective cross sections.
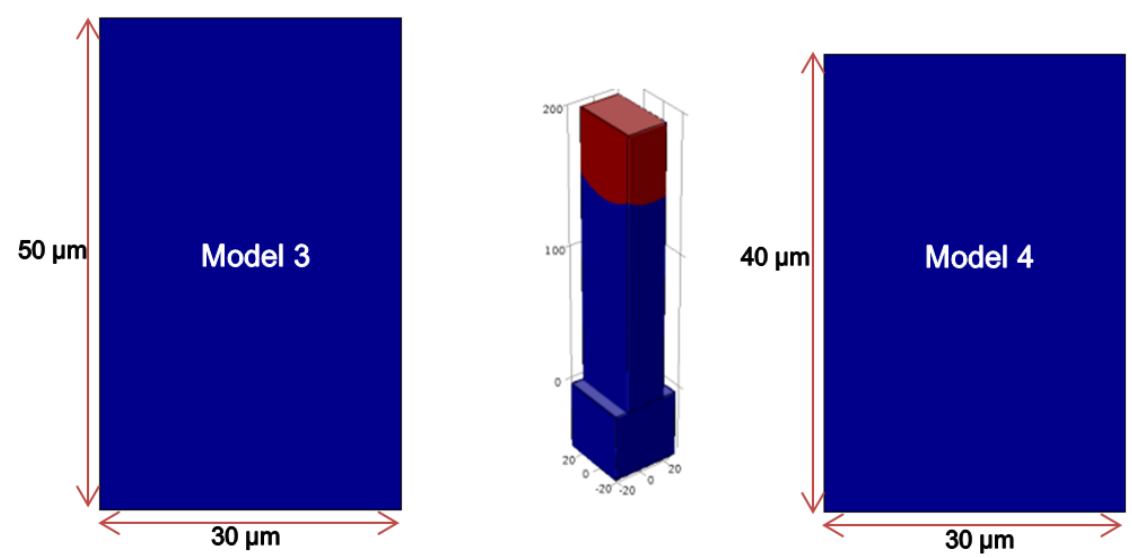

Figure 11. 3D model of the grooveless channels with their corresponding cross sections.

To examine the effect of increased contact surface between the fluid and the channel walls, the parameter $r$, which represents the surface roughness, was defined as $r=1+2 w_{p} /\left(l_{p}+d\right)$ where $w_{p}, l_{p}$, and $d$ represent the groove width, groove depth, and the distance between the grooves, respectively. According to this definition, the value of $r$ is equal to 5.3 for both Model 1 and Model 2. This means that the increase in surface resulting from addition of the grooves is identical for both models. To compare the capillary velocities in different microchannels, the vertical fluid velocity profiles at the channel inlet at $t=0 \mathrm{~s}$ were investigated for all the four models. The relevant results are shown in Figure 12. 
Model 1 velocity field $(\mathrm{m} / \mathrm{s}), \mathrm{z}$ component at $\mathrm{t}=0 \mathrm{~s}$

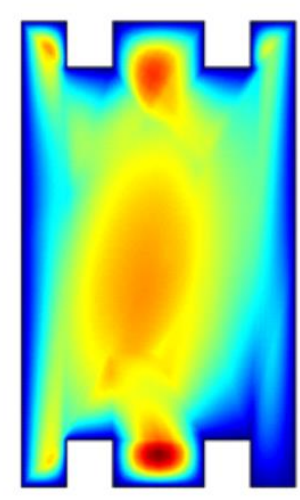

Model 2 velocity field $(\mathrm{m} / \mathrm{s}), \mathrm{z}$ component at $\mathrm{t}=0 \mathrm{~s}$
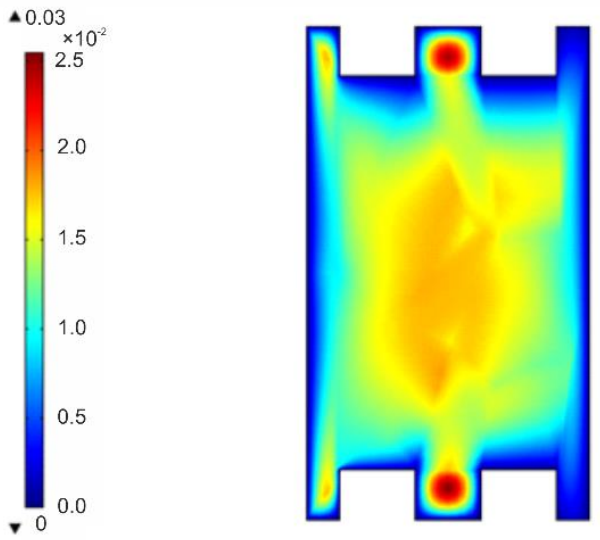

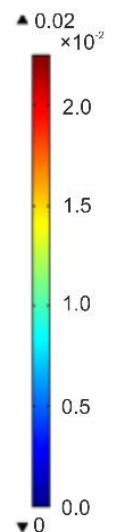

Model 3 velocity field $(\mathrm{m} / \mathrm{s}), \mathrm{z}$ component at $\mathrm{t}=0 \mathrm{~s}$
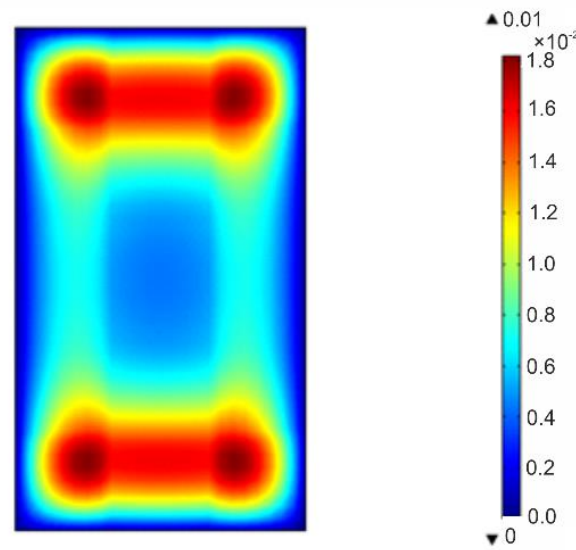

Model 4 velocity field $(\mathrm{m} / \mathrm{s}), \mathrm{z}$ component at $\mathrm{t}=0 \mathrm{~s}$
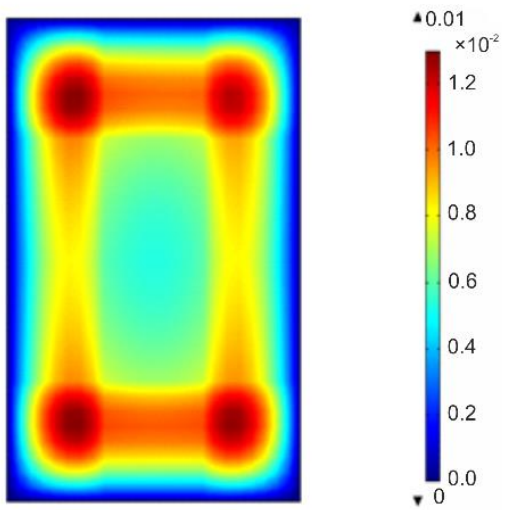

Figure 12. Vertical fluid velocity profiles obtained for the four studied models at the channel inlet at $\mathrm{t}=0 \mathrm{~s}$

In Figure 12, at the start of the capillary action, the fluid velocity reaches its maximum near the channel corners. Considering the magnitude of the fluid velocities in the four channels (Figure 12), it can be concluded that fluid velocity followed a descending trend from Model 1 to Model 4. However, to perform a more exact comparison, the capillary height variations versus time were studied for the four modelled channels. These results are shown in Figure 13. 


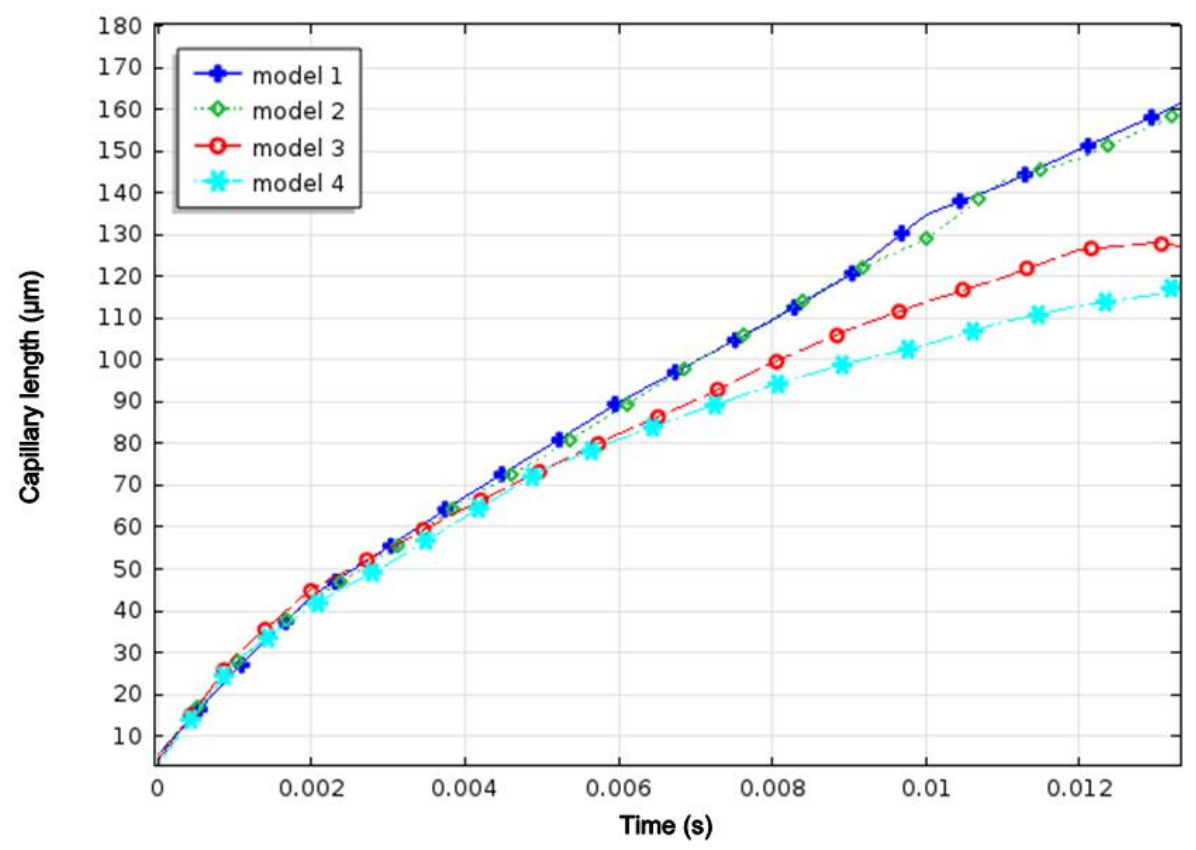

Figure 13. Capillary height versus time obtained for the four studied models.

As can be observed in Figure 13, the rate of increase in the fluid height is greater for the grooved models (as compared with the unpatterned models). However, although $r$ is equal in Models 1 and 2, in spite of the equal contact surface between the fluid and the channel walls in these two models, Model 1 is exhibited a slightly greater rate of increase in the fluid height. This is because, in addition to the fluid-wall contact surface, there is another parameter that greatly affects the velocity of the fluid. In the patterned (grooved) channel, the interface of the two fluids loses its parabolic shape, decreasing the fluid velocity. Therefore, the fluid in Model 2 moves more slowly than that in Model 1. In certain cases, this factor can even cause the fluid velocity in a grooved channel to fall below that in the ungrooved channel throughout the channel length. In other words, in the patterned (grooved) channels, two factors affect the fluid velocity: 1) contact surface between the fluid and the channel walls, and 2) the degree of deformation of the fluids interface form its original parabolic shape. The resultant of these two effects determines whether the fluid velocity increases or decreases. Figure 14 shows the velocity profile obtained for Models 1 and 3 at the channel mouth 0.01s after the start of the capillary filling. As can be observed, in the grooved channels, the vertical fluid velocity profile is less uniform than that obtained for the channel with the smooth walls. Therefore, it can be concluded that the interface of the fluids in Model 1 is less uniform than that in Model 3 , leading to a deviation from the parabolic shape of this interface and consequently, a decrease in the fluid velocity. Despite this, as the effect of increased contact surface in Model 1 is more pronounced than that of the fluid interface nonuniformity, the overall fluid velocity is greater in Model 1 as compared with Model 3. 


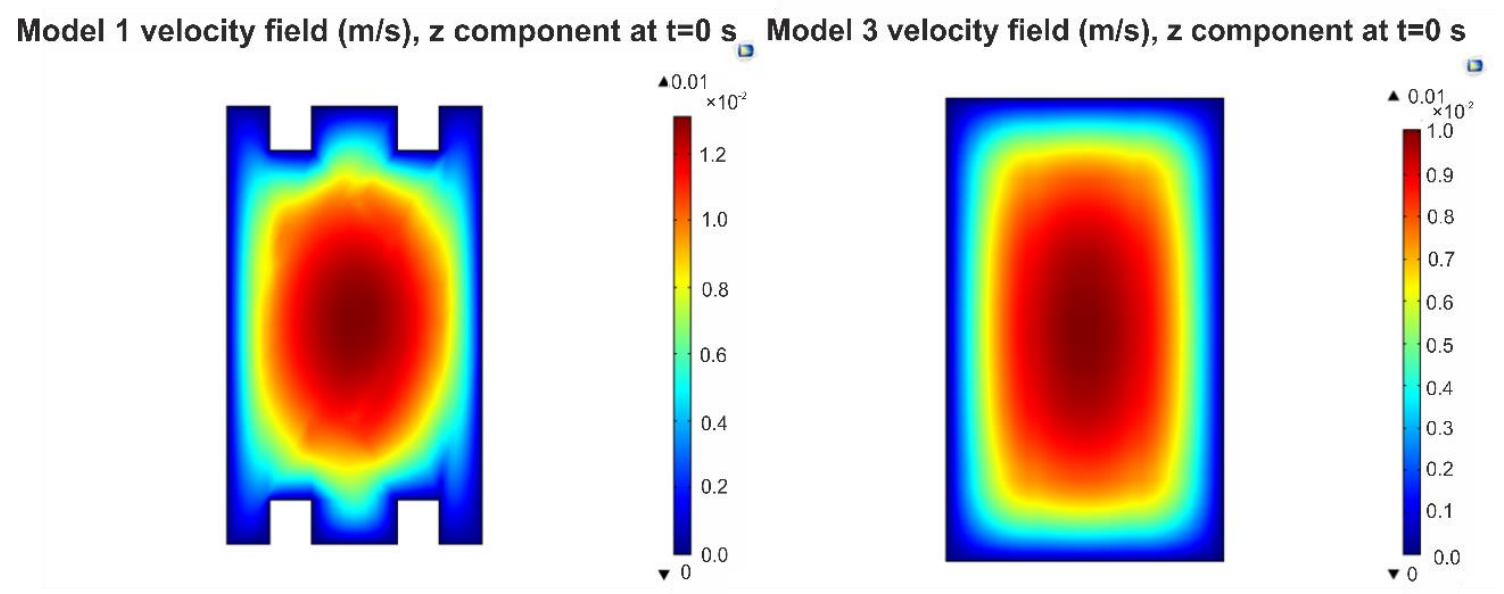

Figure 14. Fluid velocity profile at the channel mouth $0.01 \mathrm{~s}$ after start of capillary action.

\section{CONCLUSION}

As a first step in this study, the capillary filling action inside a 2D modelled channel without obstacles was modelled and the respective capillary length and velocity characteristics versus time were obtained. The fluid pressure and velocity profiles throughout the channel length during the fluid passage were also studied. It was observed that, during the capillary phenomenon, a negative pressure region developed within the fluid. This negative pressure zone is the main cause of the fluid flowing through the capillary channel. In addition, careful investigation of fluid velocity fields during the first moments of the capillary action revealed that the dynamic behaviour of the fluid was not governed by the Washburn regime but by a different regime where the capillary length is directly proportional to the square of time. By fitting a rectangular obstacle onto the inner wall of the respective microchannels, the capillary filling effect for three different fluids at contact angles of 30,50 and $60^{\circ}$ were studied, and their respective velocity versus height characteristics were obtained.

It was observed that, in accordance with the Gibbs rule, at the $30^{\circ}$ contact angle, the fluid was separated from the obstacle upon encountering it, leading to the capillary action to continue. At a contact angle of $60^{\circ}$, the fluid is remained pinned to the obstacle edge upon reaching it, and the flow was stopped. However, at a contact angle of $50^{\circ}$, the Gibbs rule was violated since, upon reaching the obstacle, the fluid was separated from it, and the flow was continued. In addition, the effect of longitudinal grooves on the capillary velocity was studied. Results showed that the grooves increase fluid velocity by $15 \%$. Finally, the effect of the grooves on the nonuniformity at the interface between the fluids was investigated. It was observed that, although adding the grooves increases the contact area between the fluid and the channel walls, the fluid velocity is decreased due to the fluids interface losing its original parabolic shape. In other words, in the patterned (grooved) channels, two factors affect the fluid velocity: 1) contact surface between the fluid and the channel walls, and 2) the degree of deformation of the fluids interface form from its original parabolic shape. The resultant of these two effects determines whether the fluid velocity increases or decreases. 


\section{REFERENCES}

[1] Majhy B, Iqbal R, Gaikwad R, Sen AK. Dynamics of capillary flow in an open superoleophilic microchannel and its application to sensing of oil. Microfluidics and Nanofluidics, 2018; 22(10): 116.

[2] Bakli C, Chakraborty S. Capillary filling dynamics of water in nanopores. Applied Physics Letters, 2012; 101(15): 153112.

[3] Acquaroli L, Urteaga R, Berli C, Koropecki R. Capillary filling in Nanostructured porous silicon. Langmuir, 2011; 27(5): 2067-2072.

[4] Nan J, Yang B. Capillary filling of Ferrofluid in homogeneous hydrophilic PDMS Microchannels. Applied Mechanics and Materials, 2015; 727: 459-464.

[5] Chakraborty S. Electroosmotically driven capillary transport of typical nonnewtonian biofluids in rectangular microchannels. Analytica Chimica Acta, 2007; 605(2): 175-184.

[6] Berli C, Urteaga R. Asymmetric capillary filling of non-newtonian power law fluids. Microfluidics and Nanofluidics, 2014; 17(6): 1079-1084.

[7] Schneider D, Valiullin R, Monson P. Filling dynamics of closed end Nanocapillaries. Langmuir, 2014; 30(5):1290-1294.

[8] Berthier J, Gosselin D, Pham A, Delapierre G, Belgacem N, Chaussy D. Capillary flow resistors: Local and global resistors. Langmuir, 2016; 32(3): 915-921.

[9] Safavieh R, Juncker D. Capillarics: Pre-programmed, self-powered microfluidic circuits built from capillary elements. Lab on a Chip, 2013; 13(21): 4180-4189.

[10] Wi Y, Kim J, Lee J, Lee J. Optimal Patterned Wettability for Microchannel Flow Boiling Using the Lattice Boltzmann Method. Coatings, 2018; 8(8): 288.

[11] Olanrewaju A, Beaugrand M, Yafia M, Juncker D. Capillary microfluidics in microchannels: from microfluidic networks to capillaric circuits. Lab on a Chip, 2018; 18(16): 2323-2347.

[12] Dewangan MK, Datta S. Flow through microchannels with topographically patterned wall: A spectral theory for arbitrary groove depths. European Journal of Mechanics-B/Fluids, 2018; 70: 73-84.

[13] Qi C, Ng CO. Rotating electroosmotic flow in a non-uniform microchannel. Meccanica, 2018; 1-16.

[14] Bui, L., Shen, Q., Hill, T., Bhuiyan, S., Barakat, R., Saavedra, V., Kong, C., Battiste, J. and Kim, Y.T., Microchannel Device for Proteomic Analysis of Migrating Cancer Cells. Biomedical Physics \& Engineering Express, 2018; 4(6): 065026

[15] Kusumaatmaja H, Pooley C, Girardob S, Pisignanob D, Yeomansa J. Capillary filling in patterned channels. Physical Review, 2008; 77(6): 067301

[16] Nayak AK, Banerjee A, Weigand B, Mixing and charge transfer in a nanofluidic system due to a patterned surface. Applied Mathematical Modelling, 2018; 54: 483-501.

[17] Chibbaro S, Costa E, Dimitrov D, Diotallevi F, Milchev A, Palmieri D, Pontrelli G, Succi S. Capillary filling in Microchannels with wall corrugations: A comparative study of the Concus-Finn criterion by continuum, kinetic, and Atomistic approaches. Langmuir, 2009; 25(21): 12653-12660.

[18] Huang W, Liu Q, Li Y. Capillary filling flows inside patterned-surface Microchannels. Chemical Engineering \& Technology, 2006; 29(6): 716-723.

[19] Das S, Waghmare PR. Early regimes of capillary filling. Physical Review, 2012; 8(6): 067301. 
[20] Olsson E, Kreiss G. A conservative level set method for two phase flow. Journal of Computational Physics, 2005; 210(1): 225-246.

[21] Concus P, Finn R. On capillary free surfaces in a gravitational field. Acta Mathematica, 1974; 132(1): 207-223.

[22] Concus P, Finn R. On capillary free surfaces in the absence of gravity. Acta Mathematica, 1974; 132(1): 177-198. 